\title{
Vaginal Chitosan Tablets with Clotrimazole-Design and Evaluation of Mucoadhesive Properties Using Porcine Vaginal Mucosa, Mucin and Gelatine
}

\author{
Emilia Szymańska, ${ }^{a}$ Katarzyna Winnicka, ${ }^{* a}$ Aleksandra Amelian, ${ }^{a}$ and Urszula Cwalina ${ }^{b}$ \\ ${ }^{a}$ Department of Pharmaceutical Technology, Medical University of Biatystok; Mickiewicza 2c, Biatystok 15-222, \\ Poland: and ${ }^{b}$ Department of Statistics and Medical Informatics, Medical University of Biatystok; Szpitalna 37, \\ Biatystok 15-295, Poland. \\ Received September 3, 2013; accepted November 7, 2013
}

Topical administration of clotrimazole represents the common use therapy in the antimycotic genitourinary tract treatment. Due to the fast self-cleaning action of the vagina, commercially available vaginal dosage forms with clotrimazole cannot assure prolonged contact time with mucosa, therefore the main objective of this study was to develop a dosage form for vaginal administration of clotrimazole using chitosan-a biodegradable and biocompatible derivative of chitin. Tablets mucoadhesive properties were examined using texture analyser under the presence of porcine vaginal mucosa and two different models of adhesive layersmucin gel and gelatine discs. In addition, friability, hardness, swelling behaviour, residence time, surface morphology of the performed tablets, the in vitro release profile of clotrimazole and clotrimazole release kinetics were determined. The release of clotrimazole from formulations with 25 or $40 \%$ of chitosan (F2 and F3) followed non Fickian diffusion through chitosan-gel layer and was retarded up to $6 \mathrm{~h}$. Additionally, tablets $\mathrm{F} 2$ showed the best results in terms of mucoadhesive properties and appeared to be a good alternative to commercially available antimycotic vaginal dosage forms.

Key words chitosan; mucoadhesive tablet; vaginal delivery; clotrimazole; texture analyser; mucoadhesion

Mycotic vaginosis (MV) is commonly occurring vaginal infection often associated with a feeling of discomfort and inconvenience. MV affects up to $75 \%$ of women at least once during their lives with a high risk of disease recurrence. ${ }^{1,2)}$ The conventional treatment of $\mathrm{MV}$ is based on combining drugs administered by both oral and vaginal routes. Vaginal drug delivery offers a great number of advantages including the hepatic first pass-effect bypass, low systemic drug exposure, ease of administration and improved bioavailability compared with the oral route. ${ }^{3)}$ For many years, imidazole derivatives like clotrimazole (CLO) (Fig. 1) have been used as the drugs of choice for the mycotic infections of the genitourinary tract treatment due to their good therapeutic responses. CLO is available in several conventional vaginal dosage forms, such as creams, ovules and tablets, ${ }^{4)}$ which do not offer prolonged delivery of the drug. Hence, it seems to be essential to develop novel formulations with increased drug residence time in the site of infection. In the recent years, the development of vaginal mucoadhesive preparations as a type of sustained release form for topical diseases treatment has gained great attention. $^{5-7)}$

Chitosan is one of the natural mucoadhesive polymers. It is a copolymer consisting of glucosamine and $\mathrm{N}$-acetylglucosamine units, which is obtained by deacetylation of chitin derived mainly from exoskeleton of crustaceans. The importance of chitosan especially in biomedicine and pharmaceutical industry has arisen from its excellent properties such as biocompatibility, biodegradability and non-toxicity. It is currently known that chitosan is biodegradaded by a number of enzymes, such as lysozyme, di- $N$-acetylchitobiase, $N$-acetylbeta-D-glucosaminidase and chitiotriosidase, which are present in human mucosa and other physiological fluids. Biodegradation of chitosan leads to the release of non-toxic oligosaccha-

The authors declare no conflict of interest.

* To whom correspondence should be addressed. e-mail: kwin@umb.edu.p rides, which can be incorporated to glycosaminoglycans and glycoproteins or be excreted. ${ }^{8-10)}$ Among polycations, it has received considerable attention for its safety-recent studies have shown that chitosan and its derivatives had no cytotoxic effect on vaginal epithelial cells. ${ }^{11,12)}$ Chitosan is also reported to show penetration enhancement properties by improving the active agent transport through epithelium layer containing tight junctions. ${ }^{12)}$ In addition, it possesses antibacterial and antifungal activity ${ }^{13)}$ and due to its good mucoadhesive properties resulted from the cationic behaviour and the presence of free hydroxyl and amine groups enabling polymer to interact with mucin by hydrogen and electrostatic bonding - chitosan is regarded as a suitable excipient to prepare buccal and vaginal dosage forms. ${ }^{14,15)}$

The aim of this work was to design mucoadhesive vaginal tablets by using chitosan as a matrix in order to improve drug residence time compared to commercially available dosage forms with CLO. The prepared tablets were analysed for weight uniformity, diameter, thickness, friability, hardness, moisture content, surface morphology, CLO content uniformity and the in vitro release. To evaluate the mucoadhesive properties of the designed tablets, swelling index, the in vitro

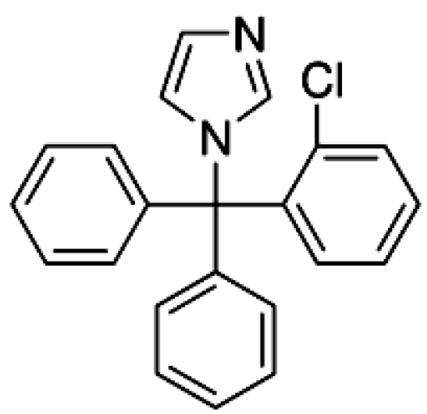

Fig. 1. Chemical Structure of CLO

(C) 2014 The Pharmaceutical Society of Japan 
residence time, maximum detachment force and work of adhesion in the presence of porcine vaginal mucosa were examined. Regarding the fact that mucoadhesive properties can be determined using different experimental conditions, ${ }^{16-18)}$ the objective of this study was also to investigate the usefulness of two different mucosa surrogates as simple adhesive models imitating vaginal mucosa-gelatin rings and mucin gel.

\section{Experimental}

Materials Chitosan (medium molecular weight (MMW), with $75-85 \%$ of deacetylation degree, viscosity of $1 \%$ solution in $1 \%$ acetic acid: $200 \mathrm{cP}$ ) (Sigma-Aldrich, Steinheim, Germany), Prosolv SMCC-50 (silicified microcrystalline cellulose) and Pruv (sodium stearyl fumarate) (JRS Pharma, Rosenberg, Germany), Tablettose 80 (spray-dried lactose) (Cardinal Pharma Ltd., Lodz, Poland), potato starch (Galfarm, Krakow, Poland) were used for tablets preparation. Clotrimazole was a gift from Ziaja Ltd. (Gdansk, Poland). Commercially available vaginal tablets with CLO (CLOTRIMAZOLUM GSK $100 \mathrm{mg}$; GlaxoSmithKline Pharmaceuticals SA, batch no: RB 2361, expiry date: 02.2014 ) were purchased locally and used as a control. Porcine vaginal mucosa from Large White Pigs weighting $\approx 200 \mathrm{~kg}$ was obtained from veterinary service of Bost slaughterhouse (Turosn Koscielna, Poland). Freshly excised tissue was washed and frozen at $-20^{\circ} \mathrm{C}$ immediately after an animal was sacrificed. ${ }^{19)}$ Samples were defrosted just before the experiment at ambient temperature and cut into $2 \mathrm{~cm}$ long and $2 \mathrm{~mm}$ thick pieces. Eighty percent acetic acid, sodium acetate, potassium dihydrogen phosphate, sodium hydroxide and disodium hydrogen phosphate were obtained from Chempur (Piekary Slaskie, Poland). Methanol was HPLC grade and it was purchased from Merck (Darmstadt, Germany). Water for HPLC was distilled and passed through a reverse osmosis system Milli-Q Reagent Water System (Billerica, U.S.A.).

Preparation of Vaginal Tablets Tablets containing $100 \mathrm{mg}$ of CLO and weighting approximately $500 \mathrm{mg}$ were prepared by direct compression method using the single punch eccentric tablet press EP-1 (Erweka, Heusenstamm, Germany) fitted with round, flat-faced $12 \mathrm{~mm}$ punches and running at a speed of $20 \mathrm{rpm}$. The blended powder was mixed in a mortar and sieved through $300 \mu \mathrm{m}$ sieve to obtain a homogenous mixture prior to compression. Preliminary experiments with constant amount of both the drug and chitosan and different excipient blends were performed to determine a suitable weight ratio of ingredients. The composition of performed formulations is shown in Table 1. The formulations F1-F4 were prepared by changing the percentage of chitosan (10-50\%) keeping on the unalterable ratio in relation to excipients used and the amount of drug.

Tablet's Physical Properties Diameter and thickness were measured using electronic calliper (Beta 1651DGT, Sovico, Italy) for 10 tablets. To study the weight uniformity, 20 tablets were weighted individually using an electronic balance (Radwag WPS 110C/10, Warsaw, Poland). Friability was determined according to $\mathrm{Ph}$. Eur. ${ }^{20)}$ by loading 20 previously weighted tablets into friabilator (Electrolab EF-1W, Mumbai, India) and set at $25 \mathrm{rpm}$ for 100 revolutions. After $4 \mathrm{~min}$, the dedusted tablets were reweighted and the percentage of weight loss was calculated. Crushing strength was analysed using a hardness tester (Dr. Schleuninger Pharmatron, Soluthurn, Switzerland) and the mean hardness was calculated. In order to determine the percentage of moisture content, accurately weighted tablets were placed on the aluminium pan of moisture analyser Radwag WPS 50SX (Warsaw, Poland), heated from 30 to $120^{\circ} \mathrm{C}$ and the average of three measurements for each batch was computed.

Drug Content Uniformity Ten randomly chosen tablets of each formulation batch were weighted accurately, powdered and dissolved in $100 \mathrm{~mL}$ of methanol in flat-bottomed flasks. The flasks were shaken for $24 \mathrm{~h}$ in a shaking water bath at $25 \pm 0.5^{\circ} \mathrm{C}$ with a rotation speed of $250 \mathrm{rpm}$ to extract the total drug contained in the tablet. The solution was then centrifuged at $4000 \mathrm{rpm}$ for $20 \mathrm{~min}$ and filtered through $0.45 \mu \mathrm{m}$ syringe nylon filters. Samples $(1 \mathrm{~mL})$ were suitably diluted with methanol and analysed for CLO content by HPLC method.

HPLC Analysis The concentration of CLO in the medium was determined by the HPLC system Agilent Technologies 1200 equipped with a G1312A binary pump, a G1316A thermostat, a G1379B degasser and a G1315B diode array detector (Agilent, Waldbronn, Germany). Data collection and analysis were performed using Chemstation 6.0 software. Isocratic separation was achieved on a Zorbax Eclipse XDB-C18, 4.6 $\times 150 \mathrm{~mm}, 5 \mu \mathrm{m}$ column (Agilent, Waldbronn, Germany). Mobile phase was methanol-phosphate buffer $\mathrm{pH}$ $7.4(4: 1, \mathrm{v} / \mathrm{v})$, the flow rate was $1.0 \mathrm{~mL} / \mathrm{min}$ and UV detection was performed at a wavelength of $210 \mathrm{~nm}^{21)}$ The column temperature was maintained at $25^{\circ} \mathrm{C}$. For injection into the HPLC system $20 \mu \mathrm{L}$ of sample was used. All reagents used for analysis were HPLC grade. The retention time of CLO was $5.2 \mathrm{~min}$. Standard calibration curve was linear over the range of $1-100 \mu \mathrm{g} / \mathrm{mL}$.

Scanning Electron Microscope (SEM) Analysis The morphology and the surface of the tablets were observed in scanning electron microscope (Hirox SH-3000 mini-SEM, Synergie 4, Lisses, France). The sample was directly fixed on a double-sided carbon tape and mounted on the Hirox

Table 1. Composition of Prepared Tablets

\begin{tabular}{|c|c|c|c|c|}
\hline \multirow{2}{*}{ Component (mg) } & \multicolumn{4}{|c|}{ Formulation } \\
\hline & $\mathrm{F} 1\left(10^{a)}\right)$ & $\mathrm{F} 2\left(25^{a)}\right)$ & $\mathrm{F} 3\left(40^{a)}\right)$ & $\mathrm{F} 4\left(50^{a)}\right)$ \\
\hline CLO & 100 & 100 & 100 & 100 \\
\hline Chitosan & 50 & 125 & 200 & 250 \\
\hline Prosolv SMCC-50 & 89.35 & 70 & 50.5 & 37.5 \\
\hline Potato starch & 63.85 & 50 & 36 & 27 \\
\hline Tablettose 80 & 191.8 & 150 & 108.5 & 80.5 \\
\hline Pruv & 5 & 5 & 5 & 5 \\
\hline
\end{tabular}

a) Percentage of chitosan. 
stage. Images were collected after 3 min pumping at $15 \mathrm{kV}$ with back-scattered electrons (BSE) detector and in the low vacuum mode.

Differential Scanning Calorimetry (DSC) DSC thermograms were performed using a differential scanning calorimeter Q2000 (TA Instruments, New Castle, U.S.A.). Samples (4-6 mg) were placed into the covered aluminium pans and heated from 30 to $280^{\circ} \mathrm{C}$ at a heating rate of $30^{\circ} \mathrm{C}$ per minute. The scans were taken under nitrogen atmosphere. An empty covered aluminum pan was used as the reference.

Swelling Index (SI) Studies Each tablet was weighted and placed separately in a $25 \mathrm{~mL}$ beaker containing $5 \mathrm{~mL}$ $0.08 \mathrm{M}$ acetic buffer $(\mathrm{pH} 4.5)$ at $37 \pm 0.5^{\circ} \mathrm{C}$. At the predetermined time intervals $(0.25,0.5,0.75,1,2,3,4 \mathrm{~h})$ tablets were removed, wiped off with filter paper and reweighted. The swelling index was calculated by using the following formula:

$$
\mathrm{SI}=\frac{W_{2}-W_{1}}{W_{1}}
$$

where SI is the swelling index, $W_{1}$ is the initial weight of the tablet, $W_{2}$ is the weight of the tablet after the particular swelling time interval. Each experiment was performed in triplicate.

In Vitro Release Study In vitro release study was carried out using USP dissolution apparatus II (Erweka Paddle Dissolution tester Type DT $600 \mathrm{HH}$, Heusenstamm, Germany). The dissolution medium was $900 \mathrm{~mL}$ of $0.08 \mathrm{M}$ acetic buffer ( $\mathrm{pH} 4.5)$ with addition of $1 \%$ surfactant maintained at $37 \pm$ $0.5^{\circ} \mathrm{C}$ and stirred at $75 \mathrm{rpm}$. Samples of $2 \mathrm{~mL}$ were withdrawn at the predetermined time intervals $(0.25,0.5,0.75,1,2,3$, $4,5,6,7 \mathrm{~h}$ ), filtered through $0.45 \mu \mathrm{m}$ cellulose acetate paper filters, diluted with methanol and analysed using the HPLC method. ${ }^{21-23)}$ Withdrawn samples were replaced with equal volumes of the fresh medium. Sink conditions were maintained throughout the experiment. Studies were conducted for six tablets from each formulation.

CLO Release Kinetics In order to investigate the kinetics governing CLO release, the obtained drug release profiles were fitted to presented below mathematical models. ${ }^{24,25)}$ Zero order equation:

$$
f_{t}=k_{0} \times t
$$

First-order equation:

$$
f_{t}=f_{t \max } \times\left(1-\exp ^{-k_{1} t}\right)
$$

Higuchi equation:

$$
f_{t}=K \sqrt{t}
$$

Korsmeyer-Peppas equation:

$$
f_{t}=k_{\mathrm{k}} \times t^{n}
$$

where $f_{t}$ is the fraction of the drug released in time $(t), k_{0}$ is the zero order kinetic constant, $f_{t \max }$ is the maximum fraction of the drug released during process, $k_{1}$ is the first order kinetic constant, $k_{\mathrm{k}}$ is constant incorporating structural and geometric dosage form, $K$ is constant reflecting the design variables of the system, $n$ is the release exponent indicating the drug re- lease mechanism.

Determination of the Residence Time The in vitro residence time of designed tablets was determined using a self-constructed apparatus (by modifying USP Disintegration tester Electrolab ED-2L, Mumbai, India), according to Nakamura et al. ${ }^{26)}$ Segments of porcine vaginal mucosa ( $2 \mathrm{~cm}$ long) were glued on the internal side of a beaker above the level of $500 \mathrm{~mL} 0.08 \mathrm{M}$ acetic buffer $(\mathrm{pH} 4.5)$ at $37 \pm 0.5^{\circ} \mathrm{C}$. After moisturizing the chitosan tablet with $100 \mu \mathrm{L}$ of acetic buffer $(\mathrm{pH}$ 4.5), a hydrated surface was put in contact with the mucosal membrane by applying a fingertip force for $30 \mathrm{~s}$. A plexiglass cylinder ( $6 \mathrm{~cm}$ diameter, weight $280 \mathrm{~g}$ ), which was vertically fixed to the apparatus was enabled to move up and down to assure the immersion of the tablet completely in the buffer solution. The time required for entire detachment of the tablet from the vaginal mucosa was noted.

Determination of the in Vitro Mucoadhesive Properties (Maximum Detachment Force and Work of Adhesion) Using Texture Analyser TA.XT.Plus Texture Analyser (Stable Microsystems, Godalming, U.K.) equipped with $5 \mathrm{~kg}$ load cell, cylinder probe and the measuring system A/MUC was used for mucoadhesion test. The in vitro mucoadhesive properties of chitosan tablets were assessed on porcine vaginal mucosa, gelatine discs and mucin gel which were placed on the platform below the texture analyser probe. Gelatine discs ( $20 \mathrm{~mm}$ in diameter, $2-3 \mathrm{~mm}$ in height) were prepared by pouring $30 \%(\mathrm{w} / \mathrm{w})$ aqueous solution of gelatine over a Petri plate and left for jellification, while $10 \%$ mucin gel was absorbed on a cellulose fiber $(20 \mathrm{~mm}$ in diameter). The tests were conducted at $37 \pm 0.5^{\circ} \mathrm{C}$. The surface of mucoadhesive material (except mucin layer) was moisturized with $100 \mu \mathrm{L}$ of $0.08 \mathrm{M}$ acetic buffer ( $\mathrm{pH} 4.5$ ). An acquisition rate of 200 points/s and a trigger force of $0.003 \mathrm{~N}$ were selected to all measurements. Each tablet was adhered to an upper probe with $\alpha$-cyanoacrylate glue and lowered onto the surface of the adhesive material with a constant speed of $0.5 \mathrm{~mm} / \mathrm{s}$. After keeping a contact time for $100 \mathrm{~s}$ under an initial contact force $(1 \mathrm{~N})$, which the parameters were determined during preliminary experiments (data not shown), the two surfaces were separated at a constant rate of $0.1 \mathrm{~mm} / \mathrm{s}$. The maximum detachment force $\left(F_{\max }\right)$ as a function of displacement was recorded directly from Texture Exponent 32 software and the work of mucoadhesion $\left(W_{\text {ad }}\right)$ expressed in $\mu \mathrm{J}$, was calculated from the area under the force versus distance curve. Commercially available tablets with CLO were used as a control. Each experiment was carried out six times.

Statistical Analysis Quantitative variables were expressed as the mean \pm standard deviation and the median. A statistical analysis was performed using nonparametric techniques: the Kruskal-Wallis and Mann-Whitney $U$-test with the Statistica 10.0 software. Differences between groups were considered to be significant at $p<0.05$.

\section{Results and Discussion}

Tablet's Physical and Chemical Properties Poor retention at the administration site of the conventional vaginal formulations with CLO and leakage caused by vertical posture results in impaired therapeutic efficiency of the drug and requires multiple daily applications. In order to overcome these limitations, it is essential to develop novel dosage forms, which ensure prolonged release of the drug in the vaginal 
cavity. ${ }^{27)}$ In the present study to obtain mucoadhesive vaginal tablets with CLO, chitosan MMW with a degree of deacetylation $75-85 \%$ was employed because of its good tableting properties and suitability for obtaining mechanically stable tablets. ${ }^{28)}$ During preliminary tableting studies with different powder blends (data not shown), a suitable type and amount of excipients were selected (Table 1). Low values of deviation from mean mass, diameter, height and hardness of the formulations F1-F3 confirmed a high uniformity of the produced tablets (Table 2). Friability and moisture's content of these formulations (less than $0.525 \%$ and $1.17 \%$, respectively) was in the acceptable range. ${ }^{20}$ Tablets F4 was mechanically unstable, which demonstrated that $50 \%$ of chitosan employed in the tablet mass was not be able to confer compactness of the formulation. However, there are reports confirmed attempts of producing tablets with even more than $80 \%$ of chitosan in a tablet, but due to different composition and destination of the designed formulations, these data are not comparable. According to obtained data of physical properties (Table 2), formulations F1-F3 were selected for further investigations.

SEM and DSC Studies To assess the surface morphology of tablets F1-F3, SEM studies were carried out. SEM photomicrographs showed heterogenous mixture of excipients with CLO crystals clearly seen inside pores, fissures and cracks. The most intact surface with a few void spaces was observed in formulation F1, whereas formulation F3 (with the highest amount of chitosan) showed the most porous structure (Fig. 2). In order to exclude possible interactions between CLO, chitosan and other excipients used, DSC analysis was conducted. Physical and mechanical properties of chitosan, which is an amorphous polymer are connected with its stretching chain and state-glassy or rubbery. Therefore, it was interesting to investigate whether the presence of crystalline CLO influenced amorphous chitosan. Pure CLO exhibited a sharp endothermic peak of $146.72{ }^{\circ} \mathrm{C}(\Delta H-667.6 \mathrm{~J} / \mathrm{g})$, corresponding to its melting point. No significant changes in CLO thermal profiles among analysed formulations were noticed, but broad endothermic peaks between 98 and $104^{\circ} \mathrm{C}(\Delta H$ in a range of -96.3 to $-241.1 \mathrm{~J} / \mathrm{g}$ ) were observed on chitosan DSC thermograms (Fig. 3). An observed shift to lower temperature and decrease in chitosan endothermic peak size in F1-F3 thermal profiles might be caused by the loss of physically adsorbed water or point on alteration the polymer state from glassy to rubbery.

SI Studies Mucoadhesion mechanism of swellable polymers is directly connected with water uptake into tablet ma- trix and gel layer formation, therefore formulations F1-F3 were submitted to swelling study in order to investigate tablet hydration capability. Adhesion occurs shortly after beginning of swelling and increases with the degree of hydration of polymer. However, excessive level of swelling reduces the mucoadhesive properties because the polymer groups necessary to link mucin chains are bound by water molecules. The swelling profiles, represented as the swelling index (SI; Eq. 1) versus time are reported in Fig. 4A. All investigated formulations' curves showed an initial rapid increase within $15 \mathrm{~min}$ due to the entry of water via metastable pores known as hysteresis of swelling mechanism ${ }^{29)}$ and reached the highest value after only $30 \mathrm{~min}$ of the study in the order F2 $>$ F1 $>$ F3. Formulation F2 (with $25 \%$ of chitosan) showed the highest swelling index (5.45), while tablets F3 with $40 \%$ of chitosan attained significantly lower value of SI (3.81). Formulation F1 registered $50 \%$ weight loss after $2 \mathrm{~h}$ as a result of tablet erosion. On the contrary, tablets F2 and F3 were compact during the study and only a slight drop in SI at the end of the test occurred. The obtained results indicate that as the concentration of chitosan increased from 10 to $25 \%$, the swelling index increased as well, but further rise in polymer concentration (up to $40 \%$ ) caused a significant decrease in water uptake. As chitosan becomes hydrated and forms a swollen gel, dissolution and surface erosion occurs simultaneously and this phenomena are slower and less intensive when higher concentrations are used. $^{30,31)}$ For this reason, tablets F2 and F3 showed higher integrity than F1.

Drug Release Studies The in vitro release profiles of CLO from formulations F1-F3 and commercially available non-modified release vaginal tablets with CLO (used as a control) are shown in Fig. 4B. Formulation F1 showed the highest initial release rate of CLO, comparable to that obtained from control (almost $100 \%$ of CLO released during the first hour of the study). The release of CLO from formulations F2 and F3 was retarded up to $6 \mathrm{~h}$ and $80 \%$ of CLO was released after 4 and $5 \mathrm{~h}$, respectively (Fig. 4B). Hydration and gel formation ability of chitosan in acidic $\mathrm{pH},{ }^{9)}$ leading to early clogging of pores in the tablet matrix hinder the entrance of water, and as a result, much slower disintegration and drug dissolution rate is observed.

In order to investigate what mechanism is responsible for prolonged CLO release, dissolution data obtained for formulations F2 and F3 were fitted to zero order, first order equations, Higuchi model (applied for matrix systems) and KorsmeyerPeppas model (employed for swellable matrices) (Eq. 2-5 in

Table 2. Physicochemical Parameters of Prepared Tablets

\begin{tabular}{lcccc}
\hline \hline & & & Formulation & \\
\cline { 2 - 5 } Parameter & F1 & F2 & F3 & F4 \\
\hline Weight (mg) & $508 \pm 5$ & $494 \pm 9$ & $497 \pm 10$ & $474 \pm 14$ \\
Deviation from average weight (\%) & 0.94 & 1.85 & 2.01 & 2.92 \\
Diameter (mm) & $12.07 \pm 0.01$ & $12.04 \pm 0.01$ & $12.07 \pm 0.01$ & $12.10 \pm 0.02$ \\
Height (mm) & $3.75 \pm 0.05$ & $3.70 \pm 0.03$ & $3.71 \pm 0.07$ & $4.08 \pm 0.03$ \\
Friability (\%) & $0.42 \pm 0.06$ & $0.30 \pm 0.08$ & $6.53 \pm 0.09$ & $3.45 \pm 0.62$ \\
Hardness (N) & $72 \pm 15$ & $66 \pm 6$ & $67 \pm 8$ & $35 \pm 8$ \\
Moisture's content (\%) & $0.25 \pm 0.11$ & $1.17 \pm 0.41$ & $0.64 \pm 0.35$ & $1.58 \pm 0.48$ \\
Drug content uniformity (\%) & $104.6 \pm 5.2$ & $97.6 \pm 6.1$ & $103.6 \pm 4.5$ & $\mathrm{nt}$ \\
\hline
\end{tabular}

a) Not tested. 
(A)

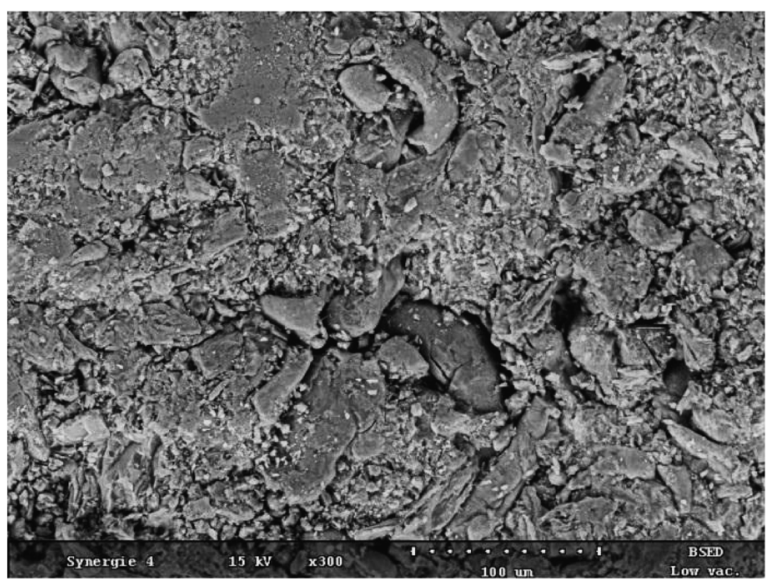

(B)

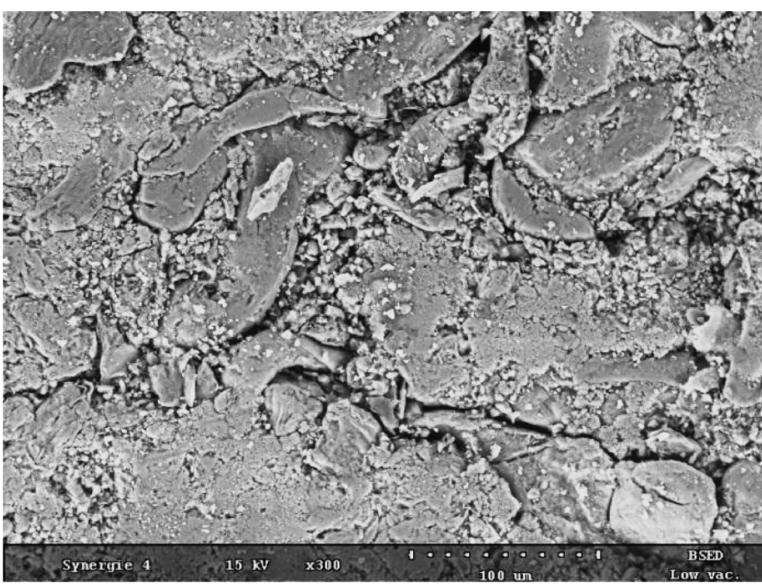

(C)

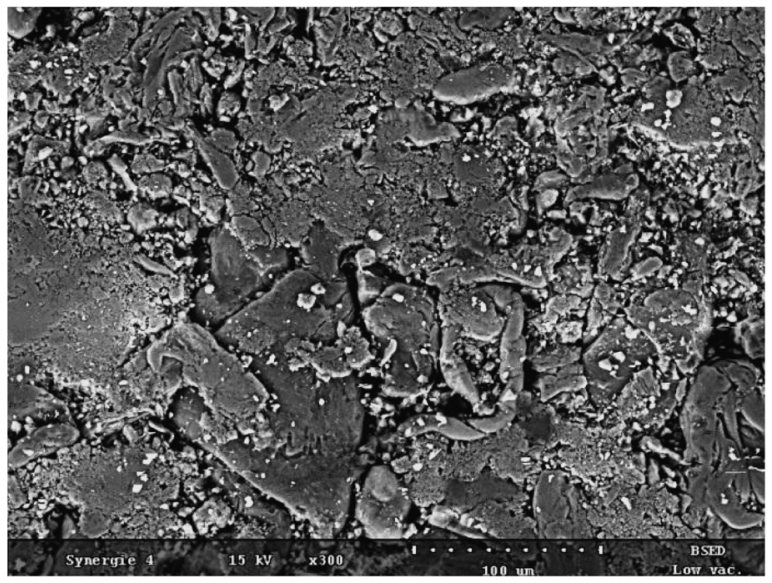

Fig. 2. Scanning Electron Micrographs of Tablets: (A) Formulation F1, (B) F2, (C) F3 (Original Magnification ×300)

Experimental). Formulation F1 was not kinetically evaluated because it was out of targeted profile limits. The calculated parameters from equations are shown in Table 3. The approximation accuracy of an individual model was defined on the basis of the regression correlation coefficient values with the acceptance of $R^{2} \geq 0.97$. The release mechanism can be controlled by polymer chain relaxation time and water penetration rate, responsible for hydration and drug diffusion. In case of cylinder shape tablets, the value of diffusional exponent $n=0.89$ means that drug release is independent of time (caseII transport), while value $n=0.45$ means that release is controlled by Fickian diffusion mechanism. A value between 0.45 and 0.89 indicates on a combination of diffusion and swelling control on release mechanism. ${ }^{24)}$ It was noticed that mechanism of CLO release from F2 and F3 did not follow case II transport but was diffusion controlled first order kinetics as the plots showed higher regression correlation coefficient (Table 3). However, based on Korsmeyer-Peppas equation, the obtained values of diffusion exponent for the formulation F2 and F3 indicated on anomalous diffusion characteristic for swelling-controlled systems. Lower value of $n$ in the case of formulation F2 (with the highest hydration level) suggests that diffusion control mechanism has the edge over the polymeric chain relaxation and may be explained by higher CLO-chitosan ratio in tablets F2. Higher amounts of CLO compared to the polymer in F2 may interfered with the chitosan macromolecular chain relaxation process, thus leading to a suppression of the relaxational mechanism. With the rise in chitosan content (F3), polymeric chain relaxation process enhanced and the $n$ value increased.

Mucoadhesion In order to investigate tablets behaviour in contact with mucosal surface and to determine residence time at the site of administration, mucoadhesive studies were performed. The in vitro residence time of designed tablets was determined using a self-constructed apparatus, according to Nakamura et $a l .{ }^{26)}$ A plexiglass cylinder, which was vertically fixed to the apparatus was enabled to move up and down to assure the immersion of the tablet completely in the $0.08 \mathrm{M}$ acetic buffer ( $\mathrm{pH} 4.5)$ solution and the time required for entire detachment of the tablet from the porcine vaginal mucosa was noted. Porcine vaginal mucosa was used for simulation of vaginal mucoadhesion due to similarity of porcine and human vaginas in terms of anatomical structure, $\mathrm{pH}$, vaginal secretion and cervical mucus production. ${ }^{32}$ Comparative studies of human and porcine vaginal mucosa revealed similarities in the morphology-both possess a nonkeratinized, stratified and squamous surface epithelium differentiated only in thickness (human: about $150-200 \mu \mathrm{m}$, porcine: $116 \mu \mathrm{m}$ ) and demonstrated the striking similarities in barrier composition and permeability. ${ }^{19)}$ Moreover, experiments with porcine mucosa application allow to replace highly expensive and requiring Bioethics Committee approval in vivo studies. It was observed that all examined tablets adhered immediately to the mucosal surface and the contact time of formulations F2 and F3 with vaginal tissue was significantly higher than control tablets (Fig. 5A) and was correlated with chitosan concentration (tablets F2 and F3 had significantly longer residence time than F1). Mechanism of chitosan mucoadhesion involves both physical and secondary chemical interactions. Physical bonds imply the entanglement followed by interpenetration of mucin chains in the swollen chitosan matrix. Second chemical interactions include hydrogen bonding and-recognised as the primary mechanism of action-electrostatic interactions between positively charged amino groups in chitosan and the negatively charged mucus gel layer. ${ }^{33)}$

The evaluation of mucoadhesive properties of tablets F1-F3 was additionally performed using texture analyser. Replacement of animal mucosa with the appropriate adhesive model can simplify the analytical procedure and improve reproducibility of the experiment, therefore the usefulness of gelatine rings and mucin gel for measurement of mucoadhesiveness of chitosan tablets were also examined. The effect of chitosan concentration on the force of detachment $\left(F_{\max }\right)$ and work 


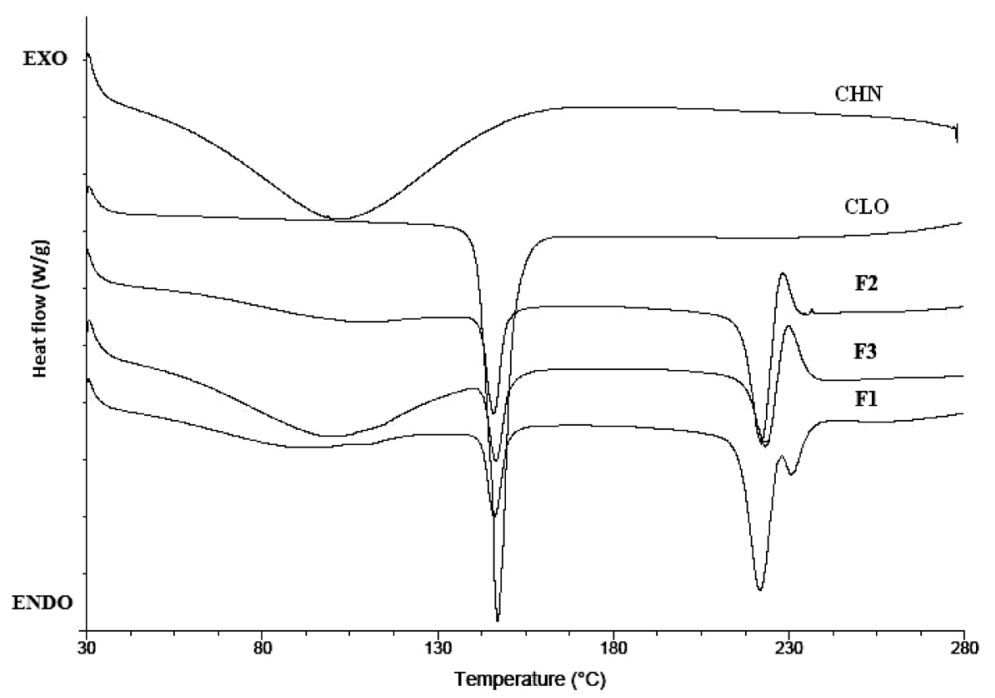

Fig. 3. DSC Thermograms of Chitosan (CHN), Clotrimazole (CLO), and Tablet Formulations F1-F3
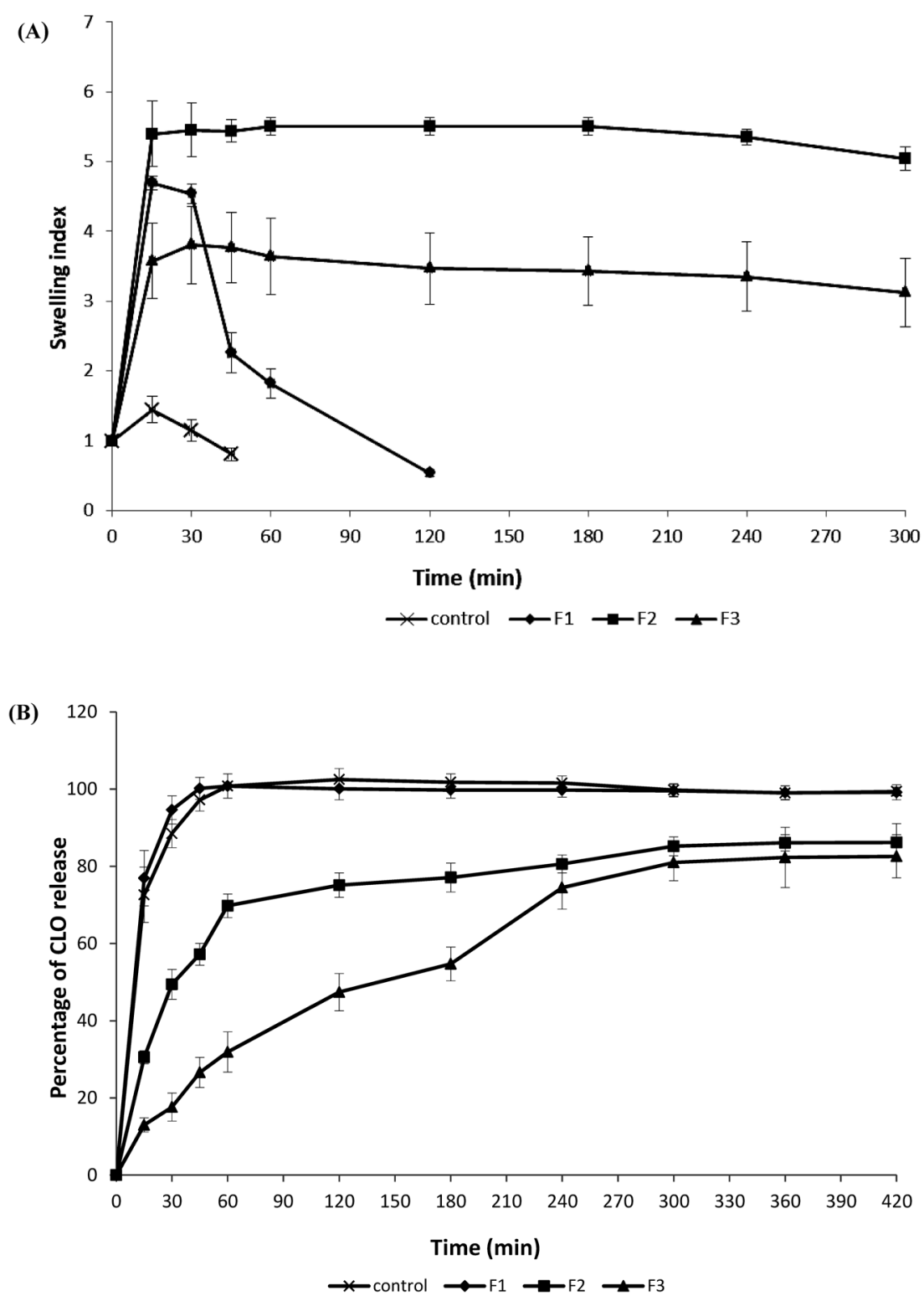

Fig. 4. Swelling Index Study of Formulations F1-F3 and Control (A) and the in Vitro CLO Release Profiles from Formulations F1-F3 and ControlCommercially Available Vaginal Tablets with CLO (B) 
Table 3. Kinetic Assessment of CLO Release from Tablets F2 and F3

\begin{tabular}{|c|c|c|c|c|c|c|c|c|c|}
\hline \multirow{3}{*}{ Formulation } & \multicolumn{9}{|c|}{ Mathematical model } \\
\hline & \multicolumn{2}{|c|}{ Zero order kinetic } & \multicolumn{2}{|c|}{ First order kinetic } & \multicolumn{2}{|c|}{ Higuchi kinetic } & \multicolumn{3}{|c|}{ Korsmeyer-Peppas kinetic } \\
\hline & $k_{0}$ & $R^{2}$ & $k_{1}$ & $R^{2}$ & $K$ & $R^{2}$ & $k_{k}$ & $R^{2}$ & $n$ \\
\hline $\mathrm{F} 2$ & 0.702 & 0.702 & 0.004 & 0.872 & 3.095 & 0.824 & 0.693 & 0.987 & 0.583 \\
\hline F3 & 0.209 & 0.955 & 0.005 & 0.979 & 4.919 & 0.987 & 0.216 & 0.984 & 0.648 \\
\hline
\end{tabular}

$k_{0}$, zero order kinetic constant; $k_{1}$, first order kinetic constant; $K$, constant reflecting the design variables of the system; $k_{k}$, constant incorporating structural and geometric dosage form; $R^{2}$, regression coefficient; $n$, the release exponent

(A)

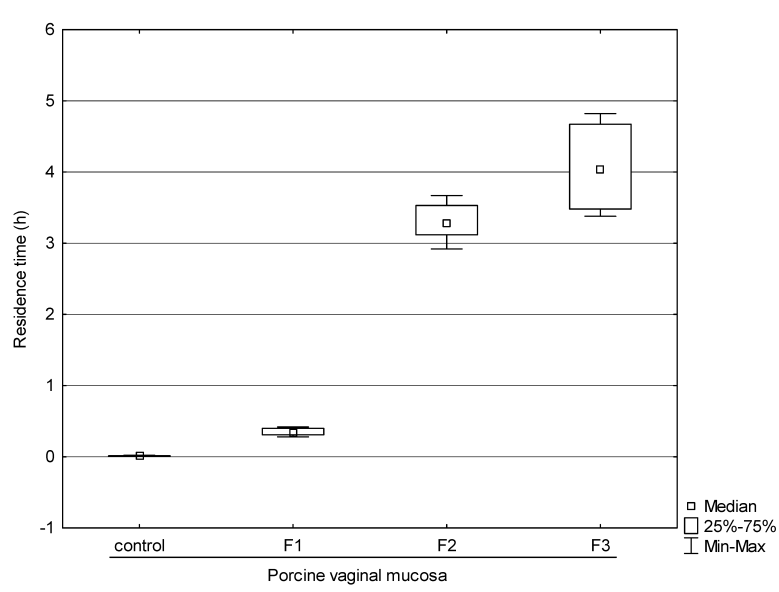

(B)

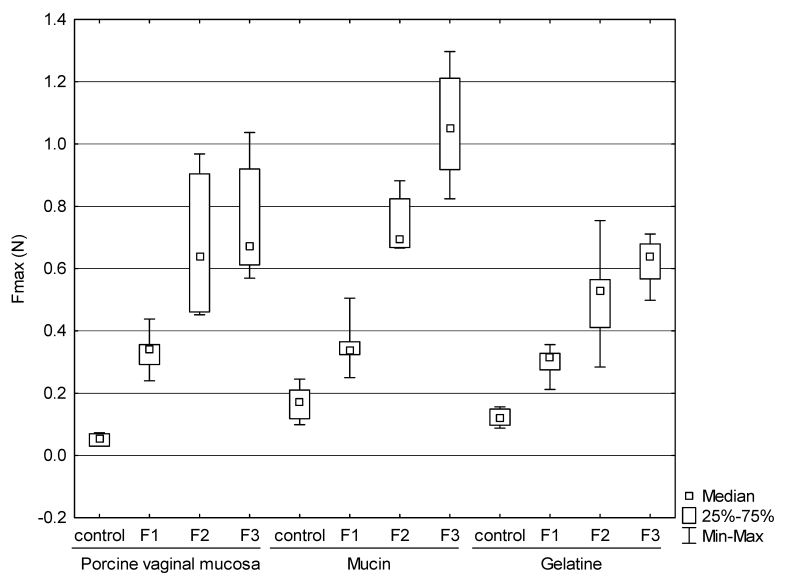

(C)

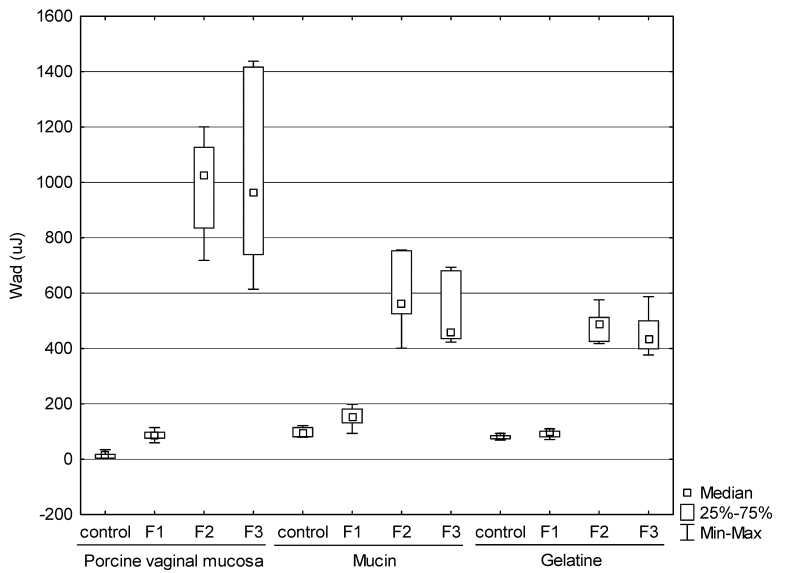

Fig. 5. Box-Plot Graphs Presenting Mucoadhesive Properties: (A) Residence Time, (B) Maximum Force of Detachment $F_{\max }$, (C) Work of Adhesion $W_{\text {ad }}$ of Formulations F1-F3 and Control (Commercially Available Tablets with CLO) Measured Using Porcine Vaginal Mucosa, Mucin and Gelatine required to overcome the tablet-adhesive material interaction $\left(W_{\text {ad }}\right)$ is presented in Figs. 5B, C. All examined tablets showed mucoadhesive properties expressed as $F_{\max }$ with the range of 0.31 to $1.04 \mathrm{~N}$ and $W_{\text {ad }}$ between 86.9 and $1021.7 \mu \mathrm{J}$. In general, the strength of mucoadhesive bond increased, by increasing the concentration of chitosan in the formulations. The highest values of $F_{\max }$ and $W_{\text {ad }}$ evaluated by using three different adhesive models were observed for formulation F3 (values obtained by using porcine vaginal mucosa: $F_{\max } 0.747 \pm 0.188 \mathrm{~N}$; median 0.671, $W_{\text {ad }} 1021.7 \pm 365 \mu \mathrm{J}$; median 961.3). It was also found that both model mucosa surrogates can be successfully used to examine the mucoadhesiveness of chitosan tablets, however gelatine ensured better correlation to porcine mucosa and ensured higher reproducibility of the experiments (Figs. $5 \mathrm{~B}, \mathrm{C})$. As $F_{\max }$ and $W_{\text {ad }}$ between F2 and F3 were not found significantly different $(p>0.05)$, a plateau in mucoadhesive properties of F2 tablets was noticed, beyond which no obvious rise in tablets mucoadhesive strength could be achieved. The obtained results were confirmed by swelling index studies (Fig. 4A) and showed a direct correlation between swelling behaviour and mucoadhesion. In formulation F3, the amount of free water available to produce swelling of mucin decreased as a result of forming compact gel-layer, thus reducing the strength of physical interactions with mucosa. Simultaneously, approximate values of mucoadhesive parameters between F2 and F3 might be explained by possible saturation of all available ionized functional groups of mucin by the positively charged chitosan, pointing on principal part of secondary chemical interactions in mucoadhesive mechanism of chitosan tablets.

\section{Conclusion}

The obtained results suggest that chitosan can be successfully used as mucoadhesive excipient for vaginal solid dosage forms with CLO. The release of CLO was depended on chitosan concentration and only tablets prepared with $25 \%$ and $40 \%$ of chitosan (F2, F3) followed the prolonged CLO release. Tablets F2 appear to be the most suitable candidate for CLO delivery with sustained release and prolonged residence time at the mucosal surface. Two different mucosa surrogatesgelatine discs and mucin gel could be successfully used to examine the mucoadhesiveness of chitosan tablets using texture analyser, however gelatine ensured better correlation to porcine mucosa and higher reproducibility of the experiments.

Acknowledgments This research was supported by Medical University of Bialystok Grant (123-15877 F). We are kindly grateful to Mr. Emilien Chutet from HIROX Synergie company (Lisses, France) for his help in taking SEM images. 


\section{References}

1) Ferrer J., Int. J. Gynaecol. Obstet., 71 (Suppl. 1), S21-S27 (2000).

2) Lanchares J. L., Hernandez M. L., Int. J. Gynaecol. Obstet., 71 (Suppl. 1), S29-S35 (2000).

3) Chien Y. W., Lee C. H., "Encyclopedia of Pharmaceutical Technology," 3rd ed. Vol. 2, Chap. Drug Delivery: Vaginal Route, ed. by Swarbrick J., Informa Healthcare, New York, 2007, pp. 1139-1361.

4) “DrugBank Database (Internet).”: 〈http//www.drugbank.ca/drugs/ DB00257), cited 24 May, 2013.

5) Perioli L., Ambrogi V., Pagano C., Scuota S., Rossi C., Int. J. Pharm., 377, 120-127 (2009).

6) Woolfson A. D., Umrethia M. L., Kett V. L., Malcolm R. K., Int. J. Pharm., 388, 136-143 (2010).

7) Perioli L., Ambrogi V., Pagano C., Massetti E., Rossi C., Colloids Surf. B Biointerfaces, 84, 413-420 (2011).

8) Kean T., Thanou M., Adv. Drug Deliv. Rev., 62, 3-11 (2010).

9) Dash M., Chiellini F., Ottenbrite R. M., Chiellini E., Prog. Polym. Sci., 36, 981-1014 (2011).

10) Muzzarelli R. A., Cell. Mol. Life Sci., 53, 131-140 (1997).

11) Meng J., Sturgis T. F., Youan B. C., Eur. J. Pharm. Sci., 44, 57-67 (2011).

12) Bonferoni M. C., Sandri G., Rossi S., Ferrari F., Gibin S., Caramella C., Eur. J. Pharm. Sci., 33, 166-176 (2008).

13) Knapczyk J., Macura A. B., Pawlik B., Int. J. Pharm., 80, 33-38 (1992).

14) Knapczyk J., Int. J. Pharm., 88, 9-14 (1992).

15) Szymańska E., Winnicka K., Acta Pol. Pharm., 69, 509-513 (2012).

16) Stasiak P., Placzek M., Lepek P., Sznitowska M., J. Dispers. Sci. Technol., 32, 1780-1785 (2011).

17) Baloglu E., Ay Senyigit Z., Karavana S. Y, Vetter A., Metın D. Y. Hilmioglu Polat S., Guneri T., Bernkop-Schnurch A., Chem. Pharm. Bull., 59, 952-958 (2011).

18) Pliszczak D., Bordes C., Bourgeois S., Marote P., Zahouani H.,
Tupin S., Mattei C. P., Lanteri P., Colloids Surf. B Biointerfaces, 92 , 168-174 (2012).

19) Squier C. A., Mantz M. J., Schlievert P. M., Davis C. C., J. Pharm. Sci., 97, 9-21 (2008).

20) "European Pharmacopeia," 6th ed., Vol. 1, Chap. 2.9, Council of Europe, Strasburg, 2007

21) Winnicka K., Sosnowska K., Wieczorek P., Sacha P. T., Tryniszewska E., Biol. Pharm. Bull., 34, 1129-1133 (2011).

22) Singh S., Jain S., Muthu M. S., Tilak R., Curr. Drug Deliv., 5 , 133-141 (2008)

23) Szymańska E., Winnicka K., Trop. J. Pharm. Res., 12, 39-44 (2013).

24) Siepmann J., Peppas N. A., Adv. Drug Deliv. Rev., 48, 139-157 (2001).

25) Costa P., Sousa Lobo J. M., Eur. J. Pharm. Sci., 13, 123-133 (2001).

26) Nakamura F., Ohta R., Machida Y., Nagai T., Int. J. Pharm., 134, 173-181 (1996).

27) Chatterjee A., Bhowmik B. B., Kumar L., J. Pharm. Res., 2, 698 700 (2009).

28) Rege P. R., Shukla D. J., Block L. H., Int. J. Pharm., 181, 49-60 (1999).

29) Wan L. S. C., Heng P. W. S., Wong L. F., Drug Dev. Ind. Pharm., 19, 1201-1210 (1993).

30) Carcía-González N., Kellaway I. W., Blanco-Fuente H., AnguianoIgea S., Delgado-Charro B., Otero-Espinar F. J., Blanco-Méndez J., Int. J. Pharm., 100, 65-70 (1993).

31) Savaşer A., Özkan Y., Işimer A., Farmaco, 60, 171-177 (2005).

32) D’Cruz O. J., Erbeck D., Uckun F. M., Toxicol. Pathol., 33, 465-476 (2005).

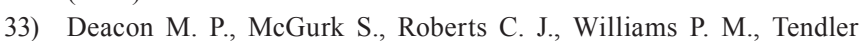
S. J., Davies M. C., Davis S. S., Harding S. E., Biochem. J., 348, 557-563 (2000) 